\title{
Possible Therapeutic Effects of Adjuvant Quercetin Supplementation Against Early-Stage COVID-19 Infection: A Prospective, Randomized, Controlled, and Open-Label Study
}

Francesco Di Pierro, (D) ${ }^{1,2}$ Giuseppe Derosa, (D) ${ }^{3,4}$ Pamela Maffioli, ${ }^{4}$ Alexander Bertuccioli, (iD ${ }^{5}$ Stefano Togni, ${ }^{6}$ Antonella Riva, (iD ${ }^{6}$ Pietro Allegrini, (ID) Amjad Khan, 7,8 Saeed Khan,' Bilal Ahmad Khan,' Naireen Altaf, ${ }^{9}$ Maria Zahid, ${ }^{9}$ Ikram Din Ujian, ${ }^{10}$ Roohi Nigar, ${ }^{1}$ Mehwish Imam Khushk, ${ }^{10}$ Maryam Phulpoto, "I Amanullah Lail, ${ }^{12}$

Bikha Ram Devrajani, ${ }^{13}$

Sagheer Ahmed ${ }^{14}$

'Scientific \& Research Department, Velleja Research, Milan, Italy; '2Digestive Endoscopy, Fondazione Poliambulanza, Brescia, Italy;

${ }^{3}$ Department of Internal Medicine and Therapeutics, University of Pavia, Pavia, Italy; ${ }^{4}$ Laboratory of Molecular Medicine, University of Pavia, Pavia, Italy; ${ }^{5}$ Department of Biomolecular Sciences (DISB), University of Urbino, Urbino, Italy; ${ }^{6}$ Indena Research and Development Department, Milan, Italy; ${ }^{7}$ Chemistry Research Laboratory, Department of Chemistry, University of Oxford, Oxford, UK; ${ }^{8}$ University of Health Sciences, Lahore, Pakistan; 'Department of Molecular Pathology, Dow International Medical College, Dow University of Health Sciences, Karachi, Pakistan; ${ }^{10}$ Department of Pathology, Liaquat University of Medical \& Health Sciences, Jamshoro, Sindh, Pakistan; "'Department of Obstetrics and Gynaecology, Liaquat University of Medical \& Health Sciences, Jamshoro, Sindh, Pakistan; ${ }^{12}$ Department of Paediatric Dow International Medical College, Dow University of Health Sciences, Karachi, Pakistan; ${ }^{13}$ Department of Medicine, Liaquat University of Medical \& Health Sciences, Jamshoro, Sindh, Pakistan; ${ }^{14}$ Shifa College of Pharmaceutical Sciences, Shifa Tameere-Millat University, Islamabad, Pakistan

Correspondence: Francesco Di Pierro Scientific \& Research Department, Velleja Research, Milan, Italy

Tel +39023510848

$\mathrm{Fax}+39023511894$

Email f.dipierro@vellejaresearch.com
Background: Quercetin, a well-known naturally occurring polyphenol, has recently been shown by molecular docking, in vitro and in vivo studies to be a possible anti-COVID-19 candidate. Quercetin has strong antioxidant, anti-inflammatory, immunomodulatory, and antiviral properties, and it is characterized by a very high safety profile, exerted in animals and in humans. Like most other polyphenols, quercetin shows a very low rate of oral absorption and its clinical use is considered by most of modest utility. Quercetin in a delivery-food grade system with sunflower phospholipids (Quercetin Phytosome ${ }^{\circledR}$, QP) increases its oral absorption up to 20 -fold.

Methods: In the present prospective, randomized, controlled, and open-label study, a daily dose of $1000 \mathrm{mg}$ of QP was investigated for 30 days in 152 COVID-19 outpatients to disclose its adjuvant effect in treating the early symptoms and in preventing the severe outcomes of the disease.

Results: The results revealed a reduction in frequency and length of hospitalization, in need of non-invasive oxygen therapy, in progression to intensive care units and in number of deaths. The results also confirmed the very high safety profile of quercetin and suggested possible anti-fatigue and pro-appetite properties.

Conclusion: QP is a safe agent and in combination with standard care, when used in early stage of viral infection, could aid in improving the early symptoms and help in preventing the severity of COVID-19 disease. It is suggested that a double-blind, placebo-controlled study should be urgently carried out to confirm the results of our study.

Keywords: SARS-CoV-2, infectious diseases, coronavirus, pneumonia, botanicals, Phytosome $\left.^{(}\right)$

\section{Introduction}

The current pandemic, known as coronavirus disease 2019 (COVID-19) triggered by the severe acute respiratory syndrome coronavirus-2 (SARS-CoV-2), is provoking devastating health, psychological and financial consequences worldwide. ${ }^{1-3}$ Although several vaccines have been developed, due to limitation in their manufacturing and distribution capacities, the desired globally "herd immunity" still appears to be a distant mirage. In addition, the constant mutations in SARS-CoV-2 put the effectiveness of these vaccination campaigns at serious risk regardless of its speed. ${ }^{4}$ The current antivirus agents being used, including hydroxychloroquine, ivermectin, lopinavir/ritonavir, have not shown any 
conclusive benefits. ${ }^{5} \mathrm{New}$ possible treatments that are safe, affordable, and worldwide available targeting the SARS-CoV -2 are therefore urgently required. ${ }^{6}$ SARS-CoV-2 proteases, like 3-chymotrypsin-like protease (3CLpro), papain-like protease (PLpro), RNA-dependent RNA polymerase, spike (S) protein and human angiotensin-converting enzyme 2 (hACE2) are considered possible targets for developing effective antiCOVID-19 drugs. ${ }^{7}$ Recently, molecular docking studies have suggested the possible binding interaction of quercetin with the 3CLpro, PLpro, and S-hACE2 complex. ${ }^{8-12}$ Some recent results, obtained by biophysical techniques, appear to support the results of the molecular docking studies. ${ }^{13}$ Quercetin, a flavonol not naturally present in the human body, is the most abundant polyphenol in fruits and vegetable and is widely used as a dietary supplement to boost the immune system and promote a healthy lifestyle. Quercetin is characterized by three crucial properties: antioxidant, anti-inflammatory and immunomodulatory. The combination of these actions allows quercetin to be a potential candidate to support all unhealthy conditions where oxidative stress, inflammation and immunity are involved. These conditions include a several disorders that can be related to cardiovascular health, healthy-aging, bones and joint health, sport and physical activity, gut, and respiratory health. $^{14}$ The above-described properties of quercetin prompted us to clinically investigate its potential therapeutic role in COVID-19. Unfortunately, as for most of polyphenols, the clinical use of quercetin has been limited due to its low solubility and reduced absorption. ${ }^{15,16}$ Recently, to improve its solid dispersion in the gut, quercetin has been formulated with sunflower lecithin. By this formulation, quercetin has shown better absorption rate, up to 20 times greater than those previously obtained with pure quercetin, without any notable side effects. ${ }^{17}$ Since a bioavailable form of quercetin could be evaluated as a potential anti-COVID-19 candidate in symptomatic patients, ${ }^{18,19}$ at the Liaquat University of Medical and Health Sciences (LUMHS), Jamshoro (Pakistan), we carried out a prospective, randomized, controlled, open-label study on 152 outpatients, not severely symptomatic at the enrolment, resulted infected with SARS-CoV-2. Our results showed a possible protective effect of bioavailable quercetin on COVID-19 patients when administered in the early days of coronavirus infection.

\section{Patients and Methods}

\section{Participants}

Between September 2020 and March 2021, 152 outpatients, with confirmed infection of SARS-CoV-2, but not with severe
COVID-19 symptoms, were enrolled at the Liaquat University of Medical and Health Sciences (LUMHS), Jamshoro (Pakistan). The inclusion criteria were to be 18 years or older of either gender, test positive for COVID-19 by polymerase chain reaction (PCR), give informed written consent to participate in the study, have oxygen saturation higher than 93\% and typical symptoms associated with COVID-19 including fever, dyspnoea, dry cough, sore throat, cold, rhinorrhoea, and conjunctivitis of mild/moderate grade to be manageable with pharmacological therapy at home. The exclusion criteria were receiving antiretroviral therapy or immune system booster medications in the last 3 months, declaring hypersensitivity or allergic reaction to quercetin or to its formula excipients, declaring glucose-6-phosphate dehydrogenase (G6PD) deficiency, being at the end stage of renal disease, and/or terminal cancer or declining to participate in the study.

\section{Study Arms, Tested Product, and Treatment Plans}

After randomization process (1:1), the 152 subjects were divided into two groups of 76 subjects each. The first group were prescribed with a standard care, to be performed at home, constituted by analgesics/anti-fevers, oral steroids, and antibiotics, as established by the hospital guidelines. This group was indicated as SC (Standard of Care). The second group were prescribed, along with the same standard care foreseen for the first group, with an adjunctive daily supplementation, lasting 30 days, constituted by 2 tablets/day (1 every 12 hours) containing quercetin formulated with sunflower lecithin in a 1:1 weight ratio. Each tablet (Quevir ${ }^{\circledR}$, a dietary supplement notified by Pharmextracta S.p.A., Italy, to the Italian Health Authorities on August 3, 2020 with document number I.5.i.h.2/ 2020/103,806) contained $500 \mathrm{mg}$ of Quercetin Phytosome ${ }^{\circledR}$ (QP) developed by Indena S.p.A., Milan, Italy. QP corresponded to $200 \mathrm{mg}$ of quercetin; therefore, each daily treatment corresponded to $400 \mathrm{mg}$ of quercetin. This group was indicated as QP. After being visited, diagnosed, and prescribed with the precise therapeutic scheme, standard care, or standard care plus QP each patient was sent back home. All enrolled patients were provided with a telephone number to be in a daily direct contact with the medical doctors involved in the study and to inform them about their medical condition. Patients were also advised to seek urgent medical assistance in case of minimal worsening or side effects occurrence. Study participants from both groups were asked not to change their usual diet and to abstain, if possible, from dietary supplements containing lactoferrin, zinc, vitamin $\mathrm{C}$ and vitamin $\mathrm{D}$ for the duration of the study. 


\section{Ethical Considerations}

In accordance with the Declaration of Helsinki, written informed consent was obtained from all participants involved in the study. The patients were assured that declining to participate in the study or leaving the study at any point would not affect the quality of their treatment and that they would thereafter receive the best care available. The study protocol was approved by the Liaquat University of Health Sciences Ethical Review Board via document number LUMHS/REC/894. The trial was registered on clinicaltrials.gov with identifier number as NCT04578158.

\section{Statistical Analysis}

To evaluate the difference in clinical outcomes, the Chi-square test (likelihood ratio and Pearson) was used. To describe the days of hospitalization versus treatment, the Box Plot was used. To compare the hospitalisation lengths the Student's $t$-test and the non-parametric Wilcoxon/Kruskal-Wallis Test (Rank Sums) procedures were used. The odds ratio between the two groups was calculated for the number of hospitalisations and for the need of non-invasive oxygen. Mean values, dispersion indexes and 95\% CI, were calculated for all continuous variables. For the analysis was used the JMP14 Pro of SAS institute (Marlow, Buckinghamshire, UK).

\section{Results}

According to the aim of our prospective, randomized, controlled and open-label study, primary endpoints were as follows: 1) need and 2) length of hospitalization, 3) need of non-invasive oxygen therapy, 4) progression to intensive care units, and 5) death. Secondary endpoints were as follows: 1) adherence, 2) tolerability, and 3) side effects as a direct consequence of the quercetin supplementation. The study has compared two groups of treatment constituted by 76 outpatients each: a Standard Care (SC) group and a second group (QP) where, besides the standard care, the subjects were treated for 30 days with two tablets/day containing as active ingredient $500 \mathrm{mg}$ of a bioavailable form of quercetin As shown in Table 1, the two groups resulted to be overlapping for numbers of patients, sex, and age stratification with no statistically significant differences. According to Table 2, outpatients needing hospitalization were 22 (28.9\%), with an average length of $6.77 \pm 3.08$ days in the SC group, and $7(9.2 \%)$ with an average length of $1.57 \pm 0.53$ in the QP group. Both data resulted to be highly significant. Patients needing not invasive oxygen therapy were 15 $(19.7 \%)$ in the SC group and $1(1.3 \%)$ in the QP group, with highly significant difference between the groups. Patients progressive to very severe conditions to proceed to intensive care unit (ICU) were $8(10.5 \%)$ in the SC group. Three of them died. No patients of the QP group proceeded to ICU and none of that group died. Both ICU and death results show significant difference between the two groups, when calculated with the likelihood ratio, and close to be significant when calculated with Pearson (Table 2).

To deeply study the positive results demonstrating the possible protective effect obtained by the add-on therapy (QP group) and to rule out a possible correlation between comorbidities and hospitalization, the possible differences in comorbidities (as declared at the enrolment) in both the groups were analysed. As shown in Table 3, the two groups had significant differences. Patients with comorbidities were 45 out of 76 in the SC group and 29 out of 76 in the QP group. Anyway, as clearly shown in Table 4, the statistical analysis performed to evaluate a possible correlation between comorbidities and hospitalization showed that the two parameters could be considered independent variables. In consequence of that, the likelihood to be hospitalized for a patient of the SC group resulted to be fourfold that of a patient of the QP group (odds ratio: 4.015873; lower 95\%: 1.597101; upper 95\%: 10.09782). To evaluate the impact of QP on otherwise healthy subjects we also analysed the two groups only considering the patients without comorbidities. As shown in Table 5, the QP group showed ameliorated clinical outcomes with a significant difference for length of hospitalization and need of oxygen therapy with a likelihood for a patient of the SC group that was 13-fold that of a patient of the QP group (odds ratio: 12.847; lower 95\%: 7.84; upper 95\%: 21.11). With regards to the number of hospitalized patients, patients with need of intensive care and deaths, the analysis showed not a statistically significant but a positive tendency for QP. This is likely due to the small number of patients considered and to the different number of comorbidity-free patients in the two groups. With regards to secondary endpoints, adherence to add-on therapy was more than $95 \%$ and quercetin was generally well tolerated with no apparent toxicity. No peculiar side effects were reported by the patients and the few cases of gastric pain and reflux, constipation, diarrhoea, meteorism, flatulence and sleep disorders were selfresolving in few days and similarly occurred in the SC group demonstrating that they likely could not be attributed to the use of quercetin (data not shown). Noteworthy, most patients of the QP group reported to investigators clear beneficial effects including reduction of fatigue and tiredness and appetite improvement (data not shown). The study was 
Table I Enrolled Outpatients According to Sex and Age Stratification

\begin{tabular}{|l|l|l|l|l|}
\hline \multicolumn{2}{|l|}{} & Group SC & Group QP & \multirow{2}{*}{} \\
\cline { 1 - 4 } N & 76 & 76 & \\
\hline \multicolumn{1}{|l|}{ Sex: Male/Female } & $46 / 30$ & $42 / 34$ & n. s. \\
\hline \multirow{2}{*}{ Age (years) } & $7 I-80$ & 2 & 1 & n. s. \\
& $6 I-70$ & 6 & 7 & n. s. \\
& $5 I-60$ & 18 & 17 & n. s. \\
& $4 I-50$ & 17 & 18 & n. s. \\
& $3 I-40$ & 14 & 13 & n. s. \\
& $21-30$ & 14 & 16 & n. s. \\
& $18-20$ & 5 & 4 & n. s. \\
\hline
\end{tabular}

Abbreviations: SC, standard care; QP, formulated quercetin (+standard care); n. s., not significant.

intended to evaluate the established primary and secondary outcomes during the 30 days from the enrolment until the end of the quercetin administration. To that date, apart from the three expired subjects of the SC group, all hospitalized patients, including those patients moved to the ICU, were discharged alive from the hospital.

\section{Discussion}

In the recent past, there have been several pandemics. Within the context of globalisation, some of these pandemics have truly raised the global risk to humankind. The COVID-19 pandemic is the latest of these. The goal of having developed several vaccines effective against SARS-CoV-2 is counteracted by the risk of constant virus mutations and by the difficulties in being quick with regards to manufacturing and distributing these vaccines worldwide. Therefore, while waiting for vaccines to get rid of this pandemic, researchers worldwide have focused all their efforts on investigating other possible treatments that are safe, affordable, and worldwide available, bearing in mind that the plant kingdom can supply chemical skeletons that, since ancient times, have provided humans with "drugs" for diverse disease conditions. In such scenario, the phytochemical agents that have demonstrated immune-modulatory, anti-viral, anti-inflammatory and antioxidant properties, along with an excellent safety profile, could be used to boost the body immune system, to reduce excessive inflammation, to inhibit viral replications and to help in preventing the COVID-19 disease development and progression. Quercetin is a phytochemical and a dietary supplement that has widely demonstrated these properties. Particularly, its antioxidant and anti-inflammatory properties seem to be closely related to its effective role against a variety of conditions related to inflammation, including viral infections, respiratory diseases, allergies, asthma, hay fever, arthritis, atherosclerosis, hypercholesterolemia, heart disease and circulatory disorders,

Table 2 Clinical Outcomes in the Two Studied Groups

\begin{tabular}{|c|c|c|c|c|c|}
\hline & Group SC & Group QP & $\mathbf{p}^{\mathbf{a}}$ & $\mathbf{p}^{\mathbf{b}}$ & $\mathbf{p}^{c}$ \\
\hline Patients hospitalized & $22(28.9 \%)$ & 7 (9.2\%) & 0.0016 & 0.0020 & \\
\hline Days of hospitalization* & $6.77 \pm 3.08$ & $1.57 \pm 0.53$ & & & 0.0001 \\
\hline Hospitalization frequencies & & & 0.0001 & 0.0037 & \\
\hline I day & 0 & 3 & & & \\
\hline 2 days & 1 & 4 & & & \\
\hline 3 days & 2 & 0 & & & \\
\hline 4 days & 3 & 0 & & & \\
\hline 5 days & I & 0 & & & \\
\hline 6 days & 3 & 0 & & & \\
\hline 7 days & 4 & 0 & & & \\
\hline 8 days & 3 & 0 & & & \\
\hline 9 days & 2 & 0 & & & \\
\hline 10 days & 2 & 0 & & & \\
\hline 16 days & I & 0 & & & \\
\hline Patients needed oxygen & 15 (19.7\%) & I (I.3\%) & 0.0100 & 0.0125 & \\
\hline Patients in ICU & $8(10.5 \%)$ & 0 & 0.0211 & 0.0608 & \\
\hline Deaths & 3 (3.9\%) & 0 & 0.04 & 0.0802 & \\
\hline
\end{tabular}

Notes: *Expressed as mean \pm standard deviation; $p^{\text {a }}$ : likelihood ratio; $p^{b}$ : Pearson; $p^{c}: t$ test and Wilcoxon/Kruskal-Wallis tests.

Abbreviations: SC, standard care; QP, formulated quercetin (+standard care); ICU, intensive care unit. 
Table 3 Number of Patients with or without Comorbidities at the Enrolment

\begin{tabular}{|l|l|l|l|l|}
\hline & $\begin{array}{l}\text { Group } \\
\text { SC }\end{array}$ & $\begin{array}{l}\text { Group } \\
\text { QP }\end{array}$ & $\mathbf{p}^{\mathbf{a}}$ & $\mathbf{p}^{\mathbf{b}}$ \\
\hline With comorbidities & $45(59.2 \%)$ & $29(38.2 \%)$ & 0.0092 & 0.0094 \\
\hline $\begin{array}{l}\text { Frequency of } \\
\text { comorbidities* }\end{array}$ & & & & \\
0 & $31(40.8 \%)$ & $47(62.8 \%)$ & & \\
\hline & $23(30.3 \%)$ & $16(22.2 \%)$ & & \\
$>1$ & $22(28.9 \%)$ & $13(15.0 \%)$ & & \\
\hline
\end{tabular}

Notes: $\mathrm{p}^{\mathrm{a}}$ : likelihood ratio; $\mathrm{p}^{\mathrm{b}}$ : Pearson; *Tuberculosis; asthma; allergy; type 2 diabetes; type I diabetes; gestational diabetes; hypertension; hypercholesterolemia; heart disease; chronic lung disease; thalassemia; polycystic ovary syndrome. Abbreviations: SC, standard care; QP, formulated quercetin (+standard care).

Table 4 Statistical Relationship Between Comorbidities and Hospitalization

\begin{tabular}{|l|l|l|l|l|}
\hline & Hospitalized & $\begin{array}{l}\text { Not } \\
\text { Hospitalized }\end{array}$ & $\mathbf{p}^{\mathbf{a}}$ & $\mathbf{p}^{\mathbf{b}}$ \\
\hline $\begin{array}{l}\text { With } \\
\text { comorbidities }\end{array}$ & $18(62.1 \%)$ & $56(45.5 \%)$ & 0.1078 & 0.1089 \\
\hline $\begin{array}{l}\text { Without } \\
\text { comorbidities }\end{array}$ & $11(37.9 \%)$ & $67(54.5 \%)$ & & \\
\hline
\end{tabular}

Notes: $p^{a}$ : likelihood ratio; $p^{b}$ : Pearson.

Abbreviations: SC, standard care; QP, formulated quercetin (+standard care).

insulin resistance and diabetes, eye-related disorders (including cataracts), stomach ulcers, cognitive impairment, gout, cancer, chronic fatigue syndrome, inflammation of the prostate, bladder, and ovaries, chronic infections of the prostate, skin disorders, including dermatitis and hives. ${ }^{14}$ Besides the "historical" pharmaco-clinical findings, the volume of data produced in the last 15 months for quercetin suggesting that this polyphenol could be a potential candidate for COVID-19, strongly prompted us to carried out a clinical study aimed to highlight its possible role within the context of this disease. Quercetin is a lipophilic compound, so it is assumed that it can cross the intestinal membranes by simple diffusion, and theoretically, this absorption is better than its glycoside forms which reach the intestines without degradation. Nevertheless, human data describe a very low oral absorption for quercetin. Due to the quercetin poor pharmacokinetics profile, it has been decided to use a lecithin delivery system form of quercetin (QP), described to be up to 20-folds more bioavailable than a not-formulated one. ${ }^{17}$ According to the kinetic parameters obtained in humans, the bioavailability rate of quercetin after the oral use of the phytosome form can be considered very far from the one considered toxic and obtained after intravenous injection in subject with cancer. ${ }^{20}$ We have then carried out a prospective, randomized, controlled and open-label study to evaluate the anti-COVID-19 effects of this orally bioavailable form of quercetin on symptomatic outpatients. According to the results of this clinical research, the add-on therapy with QP has significantly reduced the need $(-68.2 \%)$ and the length $(-76.8 \%)$ of hospitalization, the need of non-invasive oxygen therapy $(-93.3 \%$ ), the progression to intensive care units (none

Table 5 Clinical Outcomes in the Two Studied Groups Only Considering Patients with No Comorbidities at the Enrolment

\begin{tabular}{|c|c|c|c|c|c|}
\hline & Group SC & Group QP & $\mathbf{p}^{\mathbf{a}}$ & $\mathbf{p}^{\mathbf{b}}$ & $\mathbf{p}^{c}$ \\
\hline Patients & 31 & 47 & & & \\
\hline Patients hospitalized & 7 (22.6\%) & $4(8.5 \%)$ & 0.0840 & 0.0806 & \\
\hline Days of hospitalization* & $5.14 \pm 2.79$ & $1.25 \pm 0.50$ & & & 0.0097 \\
\hline Hospitalization frequencies & & & 0.0410 & 0.1147 & \\
\hline I day & 0 & 3 & & & \\
\hline 2 days & I & 4 & & & \\
\hline 3 days & 2 & 0 & & & \\
\hline 4 days & 3 & 0 & & & \\
\hline 7 days & 4 & 0 & & & \\
\hline 8 days & 3 & 0 & & & \\
\hline 9 days & 2 & 0 & & & \\
\hline Patients needed oxygen & $4(12.9 \%)$ & 0 & 0.0055 & 0.0115 & \\
\hline Patients in ICU & $2(6.5 \%)$ & 0 & 0.0521 & 0.0777 & \\
\hline Deaths & $2(6.5 \%)$ & 0 & 0.0521 & 0.0777 & \\
\hline
\end{tabular}

Notes: *Expressed as mean \pm standard deviation; $p^{\text {a }}$ : likelihood ratio; $p^{b}$ : Pearson; $p^{c}$ : $t$ test and Wilcoxon/Kruskal-Wallis tests.

Abbreviations: SC, standard care; QP, formulated quercetin (+standard care); ICU, intensive care unit. 
versus 8 subjects) and the number of deaths (none versus 3 subjects). Globally considered, the effect of QP seems to have a strong impact not only considering the difference in terms of admission to ICU and in terms of fatal outcome, but particularly considering the difference in terms of days of hospitalization. The days spent by subjects whose disease progressed and needed hospitalization were 131 for SC group and 11 for the QP one. This difference means that using QP as add-on therapy to standard care has reduced by $91.6 \%$ the days of hospitalization. Along with these results, QP not only has shown to be well tolerated and devoid of peculiar side effects, but was also reported by subjects to be beneficial against chronic fatigue and tiredness, and appetite lost, being this last two aspects commonly described as COVID-19 possibly

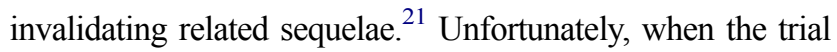
was scheduled, we did not foresee to use a standardized questionnaire to possibly evaluate these last two aspects by using a well-recognized score scale. Therefore, notwithstanding their importance, we could not quantify them. Our study has not tried to understand the possible mechanism of action of quercetin. It is suggested that this aspect should be investigated in further studies. Computational methods on molecular docking studies have apparently shown that quercetin could affect SARS-CoV-2 by interacting with 3CLpro, PLpro, and/or $\mathrm{S}$ protein. ${ }^{11-13}$ In addition to these targets, other features should be considered for quercetin as being endowed with a likely general antiviral role. Moreover, based on the strong inflammatory cascade and the blood clotting phenomena triggered during SARS-CoV-2 infection, the multifaceted aspect of quercetin, which has been well described as exerting both anti-inflammatory and thrombin-inhibitory actions should be taken into consideration. $^{22,23}$ It is well-know that P-glycoprotein (P-gp), a membrane transporter, can modify the absorption of various drugs. Anyway, pro-inflammatory cytokines released during infections have been observed to inhibit its expression and activity. ${ }^{24}$ As P-gp modifier, quercetin, by interfering with P-gp expression, could then inhibit the cytokine storm-like consequences in COVID-19 patients. $^{25}$ Quercetin is also a well-known zinc ionophore and it has been also described to cooperate with vitamin $\mathrm{C}$ in producing anti-inflammatory effects. ${ }^{26}$ Anyway, even if we cannot deny a possibly role played by these substances, naturally contained in food, we ask to the enrolled subjects to avoid the use of supplements containing them. Last, but not the least, one can assume that also the strong antioxidant role demonstrated in hundreds of in vitro and in vivo tests by quercetin ${ }^{27}$ could have favoured the positive results obtained in our study. Unfortunately, although some empirical and/or case-control clinical evaluations would seem encouraging for quercetin, high-quality clinical data to compare with do not exist and this does limit our understanding of the clinical role possibly played by this polyphenol. A randomised study performed a decade ago enrolled 1002 adult subjects affected by viral infections of the upper respiratory tract apparently showed that quercetin administered at very high dosages (1000 mg/dose) for 12 weeks reduced the days of illness in middle-aged and elderly subjects. ${ }^{28}$ More recently, an empirical study conducted at a Wuhan hospital showed that an approach where, in addition to conventional therapies patients were treated with traditional Chinese medicine remedies, including herbs with a high quercetin content, was medically safe, free from side effects, beyond those due to the conventional approach alone, and was able to improve the symptoms of patients with COVID-19. ${ }^{29}$ Besides these two pilot trials, nothing else have been published so far, at least at our knowledge. Irrespective of the randomization procedure of our study, at the enrolment the two group showed a significant difference in terms of comorbidities. Our statistical analysis suggested that comorbidities and hospitalization can be considered independent variables not directly affecting each other. Anyway, considering the importance that this aspect could have on our findings, we have re-analysed our data not considering the patients with comorbidities. The results obtained with this second approach once again demonstrated the protective role played by quercetin in the QP group. The same results were also obtained by analysing the two groups excluding comorbidity-free subjects (data not shown).

\section{Limitations}

We are perfectly aware of the pragmatic nature of our study and of the possible limits of our results, not being obtained in double-blind and in placebo-controlled conditions. For these reasons, it is suggested that these results should be urgently validated in more controlled conditions and in larger cohorts of patients. Besides these, our study also did not involve the monitoring of important inflammatory markers associated with viral infections, including C-reactive protein, D-dimer, and so on, and a repeat of RTPCR test to know exactly when the subjects became negative for SARS-CoV-2. The reason for this is that in our initial intentions we just wanted to see if quercetin, pragmatically, was able to give a real advantage in COVID-19 patients. In fact, when we designed the protocol of the study no vaccine had yet demonstrated to be safe and effective and a worldwide vaccination procedure seemed to be just a mirage. In the light of these considerations and 
limitations, we consider our results both as the first real demonstration of a possible clinical effect of quercetin in an anti-pandemic perspective and, furthermore, as a necessary starting point for a new path that must be carried out to validate our results, beyond any reasonable doubt.

\section{Conclusions}

According to the results obtained in our 30-day, prospective, randomized, controlled, open-label and pilot clinical study in which 152 COVID-19 symptomatic outpatients were enrolled, the use as adjuvant therapy of a daily dose of $1000 \mathrm{mg}$ /day of formulated quercetin (2 daily doses of $500 \mathrm{mg}$ each), corresponding to $400 \mathrm{mg}$ of quercetin delivered by sunflower lecithin (a food-grade delivery system used to improve the well-known poor oral bioavailability of quercetin), statistically improved all the clinical outcomes considered (need and length of hospitalization, need of noninvasive oxygen therapy, progression to intensive care units and death) being at the same time well tolerated by all the subjects. The clinical results obtained in our study are in line with a very recently published meta-analysis of preclinical studies where the authors concluded that the preclinical use of quercetin, or polyphenols of the quercetin type, in animal models of viral respiratory infection is able to significantly reduce: the mortality rate, the viral load, the release of proinflammatory cytokines, the presence of reactive oxygen species, the production of mucus and, therefore, also the resistance of the airways. Supplementing with quercetin-type molecules could therefore be considered a promising strategy for the treatment of viral respiratory infections. ${ }^{30}$

\section{Data Sharing Statement}

Data related to this manuscript can be made available from the corresponding author upon reasonable request.

\section{Acknowledgments}

We thank Indena S.p.A. and Pharmextracta S.p.A. for supporting the study and Prof. Martino Recchia for the execution of the statistical analysis.

\section{Disclosure}

FDP is a member of the Scientific Board of Pharmextracta. AB provided scientific advice to Pharmextracta S.p.A. PA, ST and AR are employees of and belong to the Scientific Board of Indena. AR reports a pending patent
WO2019016146A1 for quercetin phytosome. The authors report no other conflicts of interest in this work.

\section{References}

1. Kim HH, Ryu J. Social distancing attitudes, national context, and health outcomes during the COVID-19 pandemic: findings from a global survey. Prev Med. 2021;148:106544. doi:10.1016/j. ypmed.2021.106544

2. Lindinger-Sternart S, Kaur V, Widyaningsih Y, Patel AK. COVID-19 phobia across the world: impact of resilience on COVID-19 phobia in different nations. Couns Psychother Res. 2021;21(2):290-302. doi:10.1002/capr.12387

3. Nayak J, Mishra M, Naik B, Swapnarekha H, Cengiz K, Shanmuganathan V. An impact study of COVID-19 on six different industries: automobile, energy and power, agriculture, education, travel and tourism and consumer electronics. Expert Syst. 2021. doi: $10.1111 /$ exsy. 12677

4. Aschwanden C. Five reasons why COVID herd immunity is probably impossible. Nature. 2021;591(7851):520-522. doi:10.1038/d41586021-00728-2

5. Mirtaleb MS, Mirtaleb AH, Nosrati H, Heshmatnia J, Falak R, Zolfaghari Emameh R. Potential therapeutic agents to COVID-19: an update review on antiviral therapy, immunotherapy, and cell therapy. Biomed Pharmacother. 2021;138:111518. doi:10.1016/j. biopha.2021.111518

6. Karki N, Verma N, Trozzi F, Tao P, Kraka E, Zoltowski B. Predicting Potential SARS-COV-2 drugs-in depth drug database screening using deep neural network framework SSnet, classical virtual screening and docking. Int J Mol Sci. 2021;22(4):1573. doi:10.3390/ijms22041573

7. McKee DL, Sternberg A, Stange U, Laufer S, Naujokat C. Candidate drugs against SARS-CoV-2 and COVID-19. Pharmacol Res. 2020;157:104859. doi:10.1016/j.phrs.2020.104859

8. Zhang DH, Wu KL, Zhang X, Deng SQ, Peng B. In silico screening of Chinese herbal medicines with the potential to directly inhibit 2019 novel coronavirus. $J$ Integr Med. 2020;18(2):152-158. doi:10.1016/j.joim.2020.02.005

9. Smith M, Smith JC. Repurposing therapeutics for COVID-19: supercomputer-based docking to the SARS-CoV-2 viral spike protein and viral spike protein-human ACE2 interface. ChemRxiv. 2020. doi:10.26434/chemrxiv.11871402.v3

10. Williamson G, Kerimi A. Testing of natural products in clinical trials targeting the SARS-CoV-2 (Covid-19) viral spike protein-angiotensin converting enzyme-2 (ACE2) interaction. Biochem Pharmacol. 2020;178:114123. doi:10.1016/j.bcp.2020.114123

11. Pandey P, Rane JS, Chatterjee A, et al. Targeting SARS-CoV-2 spike protein of COVID-19 with naturally occurring phytochemicals: an in-silico study for drug development. J Biomol Struct Dyn. 2020:1-11. doi:10.1080/07391102.2020.1796811.

12. Khaerunnisa S, Kurniawan H, Awaluddin R, Suhartati S, Soetjipto S. Potential inhibitor of COVID-19 main protease (Mpro) from several medicinal plant compounds by molecular docking study. Preprints. 2020. doi:10.20944/preprints.202003.0226.v1

13. Abian O, Ortega-Alarcon D, Jimenez-Alesanco A, et al. Structural stability of SARS-CoV-2 3CLpro and identification of quercetin as an inhibitor by experimental screening. Int $J$ Biol Macromol. 2020;164:1693-1703. doi:10.1016/j.ijbiomac.2020.07.235

14. Anand David AV, Arulmoli R, Parasuraman S. Overviews of biological importance of quercetin: a bioactive flavonoid. Pharmacogn Rev. 2016;10(20):84-89. doi:10.4103/0973-7847.194044

15. Gao L, Liu G, Wang X, Liu F, Xu Y, Ma J. Preparation of a chemically stable quercetin formulation using nanosuspension technology. Int J Pharm. 2011;404(1-2):231-237. doi:10.1016/j. ijpharm.2010.11.009 
16. Wang W, Sun C, Mao L, et al. The biological activities, chemical stability, metabolism, and delivery systems of quercetin: a review. Trends Food Sci Technol. 2016;56:21-38. doi:10.1016/j. tifs.2016.07.004

17. Riva A, Ronchi M, Petrangolini G, Bosisio S, Allegrini P. Improved oral absorption of quercetin from quercetin Phytosome ${ }^{\circledR}$, a new delivery system based on food grade lecithin. Eur J Drug Metab Pharmacokinet. 2019;44(2):169-177. doi:10.1007/s13318-018-0517-3

18. Derosa G, Maffioli P, D'Angelo A, Di Pierro F. A role for quercetin in coronavirus disease 2019 (COVID-19). Phytother Res. 2021;35 (3):1230-1236. doi:10.1002/ptr.6887

19. Di Pierro F, Khan A, Bertuccioli A, et al. Quercetin Phytosome ${ }^{\circledR}$ as a potential candidate for managing COVID-19. Minerva Gastroenterol Dietol. 2020. doi:10.23736/S1121-421X.20.02771-3

20. Batiha GE, Beshbishy AM, Ikram M, et al. The pharmacological activity, biochemical properties, and pharmacokinetics of the major natural polyphenolic flavonoid: quercetin. Foods. 2020;9(3):374. doi:10.3390/foods9030374

21. Gautam N, Madathil S, Tahani N, et al. Medium-term outcome of severe to critically ill patients with SARS-CoV-2 infection. Clin Infect Dis. 2021. doi:10.1093/cid/ciab341

22. Almatroodi SA, Alsahli MA, Almatroudi A, et al. Potential therapeutic targets of quercetin, a plant flavonol, and its role in the therapy of various types of cancer through the modulation of various cell signaling pathways. Molecules. 2021;26(5):1315. doi:10.3390/molecules 26051315

23. Patel RV, Mistry BM, Shinde SK, Syed R, Singh V, Shin HS. Therapeutic potential of quercetin as a cardiovascular agent. Eur J Med Chem. 2018;155:889-904. doi:10.1016/j.ejmech.2018.06.053
24. Iqbal M, Ho HL, Petropoulos S, Moisiadis VG, Gibb W, Matthews SG. Pro-inflammatory cytokine regulation of P-glycoprotein in the developing blood-brain barrier. PLoS One. 2012;7(8):e43022. doi:10.1371/journal.pone.0043022

25. Pawar A, Pal A. Molecular and functional resemblance of dexamethasone and quercetin: a paradigm worth exploring in dexamethasone-nonresponsive COVID-19 patients. Phytother Res. 2020;34(12):3085-3088. doi:10.1002/ptr.6886

26. Pal A, Squitti R, Picozza M, et al. Zinc and COVID-19: basis of current clinical Trials. Biol Trace Elem Res. 2020;1-11. doi:10.1007/ s12011-020-02437-9

27. Xu D, Hu MJ, Wang YQ, Cui YL. Antioxidant activities of quercetin and its complexes for medicinal application. Molecules. 2019;24 (6):1123. doi:10.3390/molecules 24061123

28. Heinz SA, Henson DA, Austin MD, Jin F, Nieman DC. Quercetin supplementation and upper respiratory tract infection: a randomized community clinical trial. Pharmacol Res. 2010;62(3):237-242. doi:10.1016/j.phrs.2010.05.001

29. Luo E, Zhang D, Luo H, et al. Treatment efficacy analysis of traditional Chinese medicine for novel coronavirus pneumonia (COVID-19): an empirical study from Wuhan, Hubei Province, China. Chin Med. 2020;15(1):34. doi:10.1186/s13020-020-00317-x

30. Brito JCM, Lima WG, Cordeiro LPB, da Cruz Nizer WS. Effectiveness of supplementation with quercetin-type flavonols for treatment of viral lower respiratory tract infections: systematic review and meta-analysis of preclinical studies. Phytother Res. 2021. doi:10.1002/ptr.7122
International Journal of General Medicine

\section{Publish your work in this journal}

The International Journal of General Medicine is an international, peer-reviewed open-access journal that focuses on general and internal medicine, pathogenesis, epidemiology, diagnosis, monitoring and treatment protocols. The journal is characterized by the rapid reporting of reviews, original research and clinical studies

\section{Dovepress}

across all disease areas. The manuscript management system is completely online and includes a very quick and fair peer-review system, which is all easy to use. Visit http://www.dovepress.com/ testimonials.php to read real quotes from published authors. 